Aus dem anatomisch-biologischen Institut der Universität Berlin.

\title{
Selbst= und Kreuzbefruchtung bei solitären Ascidien.
}

\author{
Von
}

S. G u therz, cand. med.

Die vorliegende Untersuchung wurde im Berliner anatomischbiologischen Institut begonnen und in der zoologischen Station zu Rovigno beendigt, in der ich durch die Güte des Herrn Direktor Dr. Hermes im Herbst 1903 auf die Dauer von vier Wochen einen Arbeitsplatz erhielt und in dankenswerter Weise reichlichst mit Arbeitsmaterial versehen wurde.

Nach den Resultaten der bisherigen Untersucher kann die Frage, ob bei Ascidien Kreuz- oder Selbstbefruchtung entwicklungsfähige Embryonen liefere, nicht für alle Gattungen der Ascidien übereinstimmend beantwortet werden. Selbst für die eine Gattung Ciona sind die Angaben entgegengesetzt. K. E. v. Baer (1) nimmt bei Ciona intestinalis Selbstbefruchtung an, gestützt auf die Beobachtungen, dass Samen- und Eileiter dicht nebeneinander ausmünden, und dass durch Druck mit einer Sonde aus der Mündung des Eileiters herausgeschobene Eier im selben Moment mit Sperma übergossen werden. Kowalevsky (2) tritt bei Phallusia mammillata und Ciona intestinalis für Selbstbefruchtung ein und fügt als ihm sonderbar scheinende Beobachtung hinzu, dass bei Tieren, die einige Stunden in Gefässen mit Seewasser zugebracht hätten, die Eier zum Teil oder sämtlich ihre Entwicklungsfähigkeit verlören. v. Kupffer (3) berichtet von Ciona canina, dass ein isoliert gehaltenes Tier an vier aufeinanderfolgenden Tagen befruchtete Eier gab. Chabry (4) gibt für Ascidiella aspersa an, dass Selbst- und Kreuzbefruchtung unterschiedslos Entwicklung gäbe. Die ersten mir bekannten systematischen und unter den notwendigen Kautelen angestellten Versuche stammen von $\mathrm{Castle}(5)$ und beziehen sich auf Ciona intestinalis. Er unternahm 1. Versuche mit natürlicher Befruchtung, indem er Tiere isoliert oder $\mathbf{z u}$ zweien in sorgfältig gereinigte Glasgefässe mit frischem Seewasser brachte und zur Kontrolle nach einigen Tagen die isoliert gewesenen Tiere paarte und die gepaart gewesenen isolierte; 2. Versuche mit künstlicher Befruchtung, indem er einerseits Eier und Sperma desselben Tieres, anderseits 
Eier und Sperma zweier Tiere zusammenbrachte; um etwa anhaftende Spermatozoen abzutöten, wurden die Tiere sowie Finger und Instrumente in $90 \%$ Alkohol getaucht. Die zahlreichen Experimente führten zu dem Ergebnis, dass Selbstbefruchtung wenige (in seltenen Fällen bis $50 \%$ ) oder keine Eier zur Entwicklung bringt, während sich bei Kreuzbefruchtung die meisten (in seltenen Fällen nur 50\%, ja selbst $20 \%$ ) oder alle Eier entwickeln. Castle bemerkt ausdrücklich, dass ein derartiges Verhalten nur für Ciona sichergestellt sei, und hütet sich, seine Resultate $\mathrm{zu}$ verallgemeinern.

Meine Untersuchungen wurden an Phallusia mammillata und Ciona intestinalis angestellt, und es fand stets künstliche Befruchtung unter Entnahme der Geschlechtsprodukte aus den eröffneten Geschlechtsausführungsgängen statt. In den zur Verwendung gelangenden Tieren fanden sich fast stets Ei- und Samenleiter mit den Geschlechtsprodukten gleichmässig reichlich gefüllt. Es wurde Selbst- und Kreuzbesamung vorgenommen, und zwar gelangten bei der Kreuzbesamung in eine Schale Eier eines und Sperma eines anderen Tieres, sodass die Möglichkeit einer Selbstbefruchtung ausgeschlossen war, während bei der Vermengung der Geschlechtsprodukte zweier Tiere mit dieser Möglichkeit gerechnet werden muss. Für negativ ausfallende Besamungsversuche war es von Wichtigkeit, die normale Beschaffenheit der beteiligten Ejer und Spermatozoen zu erweisen; daher wurden stets durch gleichzeitige anderweitige Besamungen die Eier auf ihre Entwicklungsfähigkeit, das Sperma auf seine Befruchtungsfähigkeit geprüft, und es wurden solche Fälle mit negativem Ergebnis, die auf Abnormität der Eier oder Spermatozoen beruhten, natürlich ausgeschieden. Eine Reihe von Kautelen erwiesen sich als notwendig. Um unerwünschte Spermatozoen fernzuhalten, wurden Glasschalen, Instrumente, Pipetten und Finger stets sorgfältig mit Leitungswasser gereinigt; ferner wurden die Tiere vor der Eröffnung sowie noch einmal nach Freilegung der Geschlechtsausführungsgănge mit Leitungswasser abgespült; endlich fand die Besamung in Seewasser statt, das durch Erhitzen auf $60^{\circ}$ sterilisiert war und nach der Abkühlung vor der Benutzung wiederholt mit Luft geschüttelt wurde. Um unbefruchtete Eier für die Kreuzbefruchtung zu erhalten, musste die Eierentnahme der Samenentrahme vorausgehen und ein gleich- 
zeitiges Anreissen des Samenausführungsganges vermieden werden; dass wirklich keine Befruchtung stattfand, wurde dadurch bewiesen, dass ein Teil der als unbefruchtet entnommenen Eier ohne Samenzusatz aufbewahrt wurde und unentwickelt blieb. Eine weitere Kautel bei der Entnahme der Eier erwies sich durch den folgenden Umstand als notwendig. In einigen Fallen fielen die Resultate unerwarteterweise negativ aus, unter Bedingungen, unter denen die Eier der überwiegenden Mehrzahl der Versuche nach sich hätten entwickeln müssen. Dies veranlasste zu der Annahme, dass die anscheinend sämtlich zur Befruchtung reifen Eier im Eileiter der Tiere sich doch noch auf verschiedenen Entwicklungsstufen befanden, für die ein morphologisches Merkmal ohne weiteres nicht gefunden werden konnte. Gestützt wurde diese Annahme durch den bei Phallusia mammillata in einem Falle von mir erhobenen Befund, dass bei Befruchtung mit demselben Samengemische Eier aus dem Ende des Eileiters sich sämtlich entwickelten, während Eier aus der Mitte unentwickelt blieben und solche aus dem Anfang nur in geringem Prozentsatz sich entwickelten. Die sich hieraus ergebende Kautel bestand darin, dass die nur an einer Stelle des Eileiters entnommenen Eier vor der Verteilung in die Versuchsschalen erst in Seewasser gründlich durcheinander gemischt wurden. Da das Sperma bei eröffnetem Eileiter entnommen wurde, so bestand die Gefahr, den in einer Schale befindlichen Eiern bei der Besamung unerwünschte hinzuzufügen; um dies zu vermeiden, wurde das Sperma mit ganz spitzen Pipetten entnommen, die durch die Wandung des Samenleiters hindurchgestochen wurden; ausserdem wurde die das Sperma enthaltende Schale mit der Lupe auf Eier untersucht und dieselben eventuell entfernt. Für eine Versuchsreihe wurden meist drei Tiere verwandt und bei ihnen Selbstbesamung sowie alle möglichen Kombinationen von Kreuzbesamung vorgenommen, sodass, wenn die Tiere mit A, B, C, die Eier durch $q$, das Sperma durch $\delta$ bezeichnet werden, sich folgende 9 Fälle ergeben: $\mathrm{A} q \delta, \mathrm{B} q \delta, \mathrm{C} \%, \mathrm{~A} q \mathrm{~B} \delta, \mathrm{A} q \mathrm{C} \delta, \mathrm{B} q \mathrm{~A} \delta, \mathrm{B} q \mathrm{C} \delta$, $\mathrm{C} \uparrow \mathrm{A} \delta, \mathrm{C} q \mathrm{~B} \delta$. Für die Verteilung der Geschlechtsprodukte in die verschiedenen Schalen erwies sich folgendes Verfahren als zweckmässig: bei den drei Versuchstieren wurden die Geschlechtsausführungsgänge freigelegt; nunmehr wurden die zuvor etikettierten Schalen zunächst nach der in der Aufschrift be- 
zeichneten Eisorte in drei Gruppen geordnet und die Eier hineingegeben, sodann wurden sie nach der Samensorte wieder in drei Gruppen geordnet und der Samen hinzugefügt.

\section{Versuchsergebnisse bei Phallusia mammillata.}

Den Angaben meiner Versuchsergebnisse bei Phallusia ist vorauszuschicken, dass bei ihr meist ein gewisser Prozentsatz (etwa $2-15 \%$ ) von undurchsichtigen, unter dem Mikroskop braun erscheinenden Eiern auftritt, die stets unentwickelt bleiben und offenbar pathologisch verăndert sind; sie wurden bei der Feststellung des Prozentsatzes der entwickelten Eier ausser acht gelassen. Ähnliche Eier erwähnt Chabry $(4$, p. 170) bei Ascidiella aspersa. Die Entwicklung der Eier wurde bis zur Bildung von Larven verfolgt, die häufig zum Ausschlüpfen gelangten. In den Berliner Versuchen, deren Material ich aus Rovigno bezog, wurde vielfach nur Selbstbesamung vorgenommen, sodass die Zahl der Kreuzbesamungen eine geringe ist. Folgende Tabelle stellt die erhaltenen Resultate zusammen.

a) Versuche in Berlin.

\begin{tabular}{|c|c|c|c|c|c|}
\hline & $\begin{array}{c}\text { Zahl der } \\
\text { Fälle }\end{array}$ & $\begin{array}{c}\text { Entwickelte } \\
\text { Eier in } \\
\%\end{array}$ & & $\begin{array}{c}\text { Zahl der } \\
\text { Fälle }\end{array}$ & $\begin{array}{c}\text { Entwickelte } \\
\text { Eier in } \\
\%\end{array}$ \\
\hline \multirow[t]{2}{*}{$\begin{array}{c}\text { Selbst- } \\
\text { besamung }\end{array}$} & $\begin{array}{c}3 \\
2 \\
1 \\
1 \\
{[1]}\end{array}$ & $\begin{array}{r}100 \\
99 \\
92 \\
89 \\
1\end{array}$ & $\begin{array}{c}\text { Kreuz- } \\
\text { besamung }\end{array}$ & $\begin{array}{c}1 \\
1 \\
1 \\
{[1]}\end{array}$ & $\begin{array}{r}100 \\
97 \\
82 \\
0\end{array}$ \\
\hline & $7+[1]$ & & & $3+[1]$ & \\
\hline
\end{tabular}

b) Versuche in Rovigno.

\begin{tabular}{|c|c|c|c|c|c|}
\hline & $\begin{array}{c}\text { Zahl der } \\
\text { Fälle }\end{array}$ & $\begin{array}{c}\text { Entwickelte } \\
\text { Eier in } \\
\%\end{array}$ & & $\begin{array}{c}\text { Zahl der } \\
\text { Fälle }\end{array}$ & $\begin{array}{c}\text { Entwickelte } \\
\text { Eier in } \\
\% / 1\end{array}$ \\
\hline \multirow[t]{2}{*}{$\begin{array}{c}\text { Selbst- } \\
\text { besamung }\end{array}$} & $\begin{array}{c}20 \\
t \\
1 \\
{[1]} \\
{[1]} \\
{[1]}\end{array}$ & $\begin{array}{c}100 \\
98-94 \\
77 \\
10 \\
6 \\
0\end{array}$ & $\begin{array}{c}\text { Kreuz- } \\
\text { besamung }\end{array}$ & $\begin{array}{r}27 \\
2 \\
3 \\
1 \\
{[1]} \\
{[8]}\end{array}$ & $\begin{array}{c}100 \\
97-95 \\
92-90 \\
50 \\
12 \\
\text { 0 od. fast 0 }\end{array}$ \\
\hline & $25+[3]$ & & & $33+[9]$ & \\
\hline
\end{tabular}


Die eingeklammerten Zahlen beziehen sich auf solche Besamungen mit negativem oder nahezu negativem Ausfall, bei denen die obenerwähnte Kautel der Durchmischung der Eier vor der Verteilung in die einzelnen Schalen noch nicht zur Anwendung gelangte. Diese Fälle beruhen höchstwahrscheinlich auf der nicht völligen Reife der in den Versuchen verwandten Eier, wofür die bereits mitgeteilte Beobachtung spricht, dass in den verschiedenen Teilen des Eileiters Eier von verschiedener Befruchtungsfähigkeit vorkommen können. In den vier Fällen von negativem Ergebnis der Selbstbesamung erbielt ich ausserdem nach 24 stündigem Liegenlassen der eröffneten Tiere auf Eis Entwicklung sämtlicher entnommener Eier bei Selbstbesamung, was ich anfangs als eine Wirkung des 24 stündigen Aufbewahrens auf die Neigung der Eier zur Selbstbefruchtung ansah, jetzt aber auf die spätere Entnahme reiferer oder durch das Aufbewahren nachgereifter Eier beziehen möchte. In den zehn Fällen von negativem Ergebnis der Kreuzbesamung brachte die gleichzeitig vorgenommene Selbstbesamung sämtliche Eier zur Entwicklung, und die negativen Fälle verteilten sich so, dass nicht etwa gewisse Eier auf Selbstbesamung positiv, auf Kreuzbesamung negativ reagierten, sondern so, dass die Eier auf Kreuzbesamung in dem einen Falle positiv, im anderen negativ reagierten. Schon dieser Umstand legte den Verdacht nahe, dass es sich um Eier von nicht genügender Reife handle. Unter 18 in obiger Tabelle inbegriffenen Fällen von Kreuzbesamung, in denen die Kautel der Durchmischung der Eier vor der Verteilung in die Schalen innegehalten wurde, ergaben 16 alle Eier, einer fast alle, einer $50 \%$ der Eier entwickelt. So glaube ich also sagen zu können, dass bei Phallusia mammillata Selbstbefruchtung sowohl wie Kreuzbefruchtung gleichmässig gute Entwicklung gibt.

\section{Versuchsergebnisse bei Ciona intestinalis.}

Versuche an Ciona wurden nur in Rovigno angestellt, und es wurde hierbei von vornherein die Kautel der Durchmischung der Eier vor der Verteilung in die Schalen angewandt. Die Entwicklung der Eier wurde bis zum Ausschlüpfen lebhaft beweglicher Larven verfolgt. Die Resultate der Besamungen waren die folgenden: 


\begin{tabular}{|c|c|c|c|c|c|}
\hline & $\begin{array}{l}\text { Zahl der } \\
\text { Fälle }\end{array}$ & $\begin{array}{c}\text { Entwickelte } \\
\text { Eier in . } \\
0 \% 0\end{array}$ & & $\begin{array}{c}\text { Zahl der } \\
\text { Fälle }\end{array}$ & $\begin{array}{c}\text { Entwickelte } \\
\text { Eier in } \\
\%\end{array}$ \\
\hline \multirow[t]{2}{*}{$\begin{array}{c}\text { Selbst- } \\
\text { besamung }\end{array}$} & $\begin{array}{l}8 \\
1 \\
1\end{array}$ & $\begin{array}{r}0 \\
8 \\
13\end{array}$ & $\begin{array}{c}\text { Kreuz- } \\
\text { besamung }\end{array}$ & $\begin{array}{r}10 \\
6 \\
1 \\
1 \\
1 \\
1\end{array}$ & $\begin{array}{c}100 \\
\text { fast } 100 \\
90 \\
85 \\
58 \\
15\end{array}$ \\
\hline & 10 & & & 20 & \\
\hline
\end{tabular}

Die in zwei Fallen durch Selbstbefruchtung in geringem Prozentsatz zur Entwicklung gebrachten Eier ergaben langgestreckte lebhaft bewegliche Larven, die sich in nichts von den durch Kreuzbefruchtung erzeugten unterschieden. In den beiden Fällen von Kreuzbesamung bei Ciona mit 58 und $15 \%$ entwickelten Eiern, sowie in dem Falle von Kreuzbesamung bei Phallusia mit $50 \%$ scheinen, falls man nicht Versuchsfehler annehmen will, Fälle vorzuliegen, in welchen die Kreuzbesamung zwischen gesunden Eiern und Spermatozoen zum Teil oder fast ganz versagt. Versuche, künstlich eine Selbstbefruchtung bei Ciona zu bewirken (durch Aufbewahren der Eier in Seewasser, Aufbewahren eröffneter Tiere auf Eis, Einwirkung einer Temperatur von $26-29^{\circ} \mathrm{C}$.), habe ich nur in geringer Menge und mit negativem Ergebnis angestellt. Dass durch die Selbstbesamung die Eier weder befruchtet noch geschädigt werden, geht aus der von mir gemachten Erfahrung hervor, dass Eier, die einer Selbstbesamung ausgesetzt gewesen waren und einige Stunden später einer Kreuzbesamung. unterworfen wurden, sich normal entwickelten. Meine Ergebnisse bei Ciona, dass Selbstbesamung negativ, Kreuzbesamung positiv ausfällt, stimmen mit denen $\mathrm{Castles}$ vollkommen überein.

Zusammenfassend ist zu sagen, dass Selbstbesamung bei Phallusia alle oder fast alle Eier, bei Ciona keine Eier oder nur einen geringen Prozentsatz zur Entwicklung bringt. Kreuzbesamung bringt bei beiden Tieren alle oder fast alle Eier zur Entwicklung.

Wie die Befruchtung unter natürlichen Bedingungen vor sich geht, lässt sich bei Ciona leicht vorstellen: Kreuzbefruchtung muss, wie Castle sagt, die Regel, Selbstbefruchtung die Ausnahme 
sein. Für den späteren Verlauf der Entwicklung ist die Möglichkeit in Betracht zu ziehen, dass die aus Selbstbefruchtung hervorgegangenen wenigen Larven in der Entwicklung stehen bleiben und so ausgemerzt werden. Schwieriger gestaltet sich die Beurteilung der natürlichen Verhältnisse bei Phallusia. Von Bedeutung ist hier die Art und Weise, wie die Geschlechtsprodukte entlassen werden, worüber für Ciona intestinalis und Molgula Manhattensis Beobachtungen Castles (5) vorliegen. Täglich zu bestimmter Zeit, gegen Tagesanbruch, sieht man die Tiere zwei- oder dreimal sich heftig zusammenziehen und dann wieder ihre gewöhnliche Ruhe annehmen, worauf man die abgelegten Eier im Aquarium vorfindet. Hiernach scheint es sich um die gleichzeitige Ausstossung von Eiern und Spermatozoen zu handeln, die also in der Kloake einer gründlichen Durchmischung ausgesetzt sein würden. v. Ku p ffer (3) ist der Meinung, dass bei Ciona canina zweifellos eine Mengung beider Geschlechtsprodukte in der Kloake erfolge, da ein Häufchen frisch ausgestossener Eier, mit der Pipette vom Boden des Gefässes auf den Objektträger gebracht, stets zahlreiche Spermatozoen an den Zotten der Eihaut zeige. Ist der Ausstossungsmodus bei Phallusia ein ähnlicher, was der Untersuchung bedarf, so müsste man als normal Selbstbefruchtung annehmen, denn die Zeit der Durchmischung der Geschlechtsprodukte in der Kloake genügt wohl, um in jedes Ei ein Spermatozoon eindringen zu lassen; würden Eier und Sperma zu verschiedenen Zeiten ausgestossen, so wäre die Möglichkeit für Kreuz- und Selbstbefruchtung gegeben und durch Absterben der aus Selbstbefruchtung hervorgegangenen Larven könnte die Kreuzbefruchtung das Übergewicht gewinnen. Erwähnt sei noch die Möglichkeit, dass mit dem Atemwasser Spermatozoen von aussen in die Kloake gelangen und beim Austreten der Eier aus dem Ovidukt Kreuzbefruchtung bewirken könnten; eine Möglichkeit, die man ausschliessen könnte, wenn tatsächlich nur alle 24 Stunden eine Ausstossung der Geschlechtsprodukte stattfände und wenn die Spermatozoen von Phallusia sich in Seewasser nur kürzere Zeit als 24 Stunden am Leben erhielten, was mir nach gelegentlichen Beobachtungen wahrscheinlich ist.

Man könnte daran denken, den auffälligen Gegensatz im Verhalten von Phallusia und Ciona bezüglich der Selbstbesamung 
mit ihrer verschiedenen Lebensweise in Verbindung zu bringen. Phallusia findet sich fast stets als Einzeltier, Ciona dagegen in Gruppen von wenigen bis sehr zahlreichen Tieren, deren Cellulosemäntel gegen die Basis zu miteinander verwachsen sind. Man könnte nun meinen, dass für Phallusia die Gelegenheit zur Kreuzbefruchtung geringer sei als für Ciona. Indessen kommen die Phallusien, wie mir Herr Dr. Hartme yer gütigst mitteilte, auf dem Meeresgrunde in betrăchtlicher Zahl nahe beieinander vor, was aus der reichen Ausbeute beim Dredgen hervorgehe, sodass eine geringere Gelegenheit zur Kreuzbefruchtung bei Phallusia nicht anzunehmen sei. Übrigens finden sich gelegentlich auch Phallusien mit den Mänteln verwachsen; einen derartigen Fall, in dem zwei Tiere mit der ganzen Ventralseite zusammenhangen, weist das Berliner zoologische Museum auf, einen anderen, in dem sich die Verwachsung zweier Tiere auf die Gegend der Basis beschränkte, hatte ich selbst zu beobachten Gelegenheit.

Eine kurze Betrachtung der Befruchtungsverhältnisse bei den übrigen Tunikaten sei angefügt. Bei den Salpen und im allgemeinen auch bei den Appendikularien ist das Verhalten ein eindeutiges: es hat ausgesprochene Dichogamie statt und zwar bei den Salpen als Protogynie (indem sämtliche Glieder einer Kette ihre Geschlechtsorgane im gleichen Sinne ausgebildet zeigen), bei den Appendikularien als Protandrie. Bei den letzteren erwähnt Seeliger (Bronns Klassen und Ordnungen des Tierreichs, III. Suppl., 1895-1903) zwei abweichende Beobachtungen: bei Kowalevskia solle der Zeitunterschied zwischen dem Eintritt der männlichen und dem der weiblichen Geschlechtsreife nur wenige Minuten betragen, und da Spermatozoen und Eier hier in die Leibeshöhle entleert würden, so könnte die Selbstbefruchtung kaum sicher vermieden werden; auch habe er bei Fritillaria furcata reife Eier und entleerte Spermatozoen gleichzeitig in der Leibeshöhle des Elterntieres angetroffen. Ein Schluss auf die Möglichkeit des Zustandekommens der Selbstbefruchtung ist aus diesen Beobachtungen nicht zu machen. Bei den Pyrosomen herrscht Protogynie; die einzelnen Individuen sind von ungleichem Alter und in verschiedenen Stadien der Ausbildung der Geschlechtsdrüsen, sodass in einer Kolonie, allerdings in getrennten Individuen, gleichzeitig reife Eier und Spermatozoen vorhanden sein können. Das Verhalten bei den Synascidien ist nach Seeligers Zusammen- 
stellung (1. c.) bei den verschiedenen Gattungen und selbst bei den Arten ein sehr verschiedenartiges. Einerseits finden sich vielfach typische Hermaphroditen, anderseits ist das Vorkommen rein diöcischer Formen behauptet worden. Zwischen diesen beiden Extremen stehen die häufigen Fälle von Dichogamie ${ }^{1}$ ), die wiederum sowohl als Protandrie - wobei die anfänglich männlichen Tiere meist später zu typischen Zwitterformen werden - wie auch als Protogynie auftreten kann; auch bei dieser führen wohl die meisten Tiere, wenigstens wăhrend einer kurzen Lebensperiode, einen in beiden Abschnitten tätigen Zwitterapparat. Bei den zu dieser letzten Gruppe gehörigen Botrylliden entwickeln sich die Hoden erst so spät, dass eine Selbstbefruchtung ausgeschlossen erscheint; da nun die mit Geschlechtsorganen ausgestatteten, durch Knospung entstandenen Tiere wieder Knospen entwickeln, in denen zuerst die Eier und später die Hoden reifen, so kann der Fall eintreten, dass die Zeit der Eireife des Tochtertiers mit der Produktion reifer Spermatozoen im Muttertier zusammenfallt und die Gelegenleit zur Inzucht gegeben ist. Bei der Mehrzahl der von Hartmeyer (6) behandelten Polyclinidenarten aus den Gattungen Amaroecium, Aplidium und Synoecum findet sich, wie im besonderen aus den Abbildungen zu entnehmen ist, die eine der beiden Gonaden stärker entwickelt als die andere, ein Verhalten, das sämtliche Individuen einer Kolonie in gleicher Weise zeigen; eine Spezies mit gleichmässiger Ausbildung beider Geschlechtsdrüsen ist eine Seltenheit.

Direkte Analogien $\mathrm{zu}$ den im vorstehenden mitgeteilten Versuchsergebnissen finden sich im Ptlanzenreiche. Es sind nämlich Fälle bekannt, in denen nahe verwandte Pflanzen sich bezüglich der Frage der Selbst- oder Kreuzbefruchtung ganz verschieden verhalten. Nach $W$ iesner (Elemente der wissenschaftlichen Botanik III, 1902) seien einige derartige Fälle angeführt. Roggen ist selbst-steril, d. h. der Pollen wirkt in der eigenen Blüte nicht befruchtend; Gerste ist dagegen in der Regel auf Selbstbefruchtung angewiesen, ja wenn zur Zeit des Öffnens der Antheren nicht eine bestimmte Temperatur erreicht wird, so erfolgt sogar die Befruchtung in der geschlossenen Blüte. Die unscheinbare nur selten von Insekten besuchte Viola arvensis

1) Auch bei Monascidien findet sich Dichogamie (Seeliger, 1. c.) 
120 S. Gutherz: Selbst- und Kreuzbefruchtung bei solitären Ascidien.

liefert bei Eigenbefruchtung keimfähigen Samen; die grossblütige häufig von Insekten aufgesuchte Viola tricolor bringt nur durch Wechselbefruchtung keimfähigen Samen hervor. Für Brassica oleracea wurde konstatiert, dass sowohl Selbstbefruchtung als Kreuzung zur Ausbildung reichlicher keimfähiger Samen führt, wahrend Brassica campestris nur durch Wechselbefruchtung ein günstiges Befruchtungsresultat liefert. Von Interesse ist es, dass Pflanzen vorkommen, die nur kleistogame Blüten hervorbringen, deren geschlechtliche Fortpflanzung also ausschliesslich durch Selbstbefruchtung erfolgt.

Zum Schlusse ist es mir eine angenehme Pflicht, Herrn Geheimrat 0. Hertwig, der mir dieses Thema stellte, hierfür und für sein liebenswürdiges Interesse am Verlaufe der Arbeit, sowie Herrn Dr. G. Wetzel für mannigfachste Unterstützung und Beratung meinen ergebenen Dank auszusprechen.

\section{Literatur.}

1. K. E.v. Baer: Entwickeln sich die einfachen Ascidien in der ersten Zeit nach dem Typus der Wirbeltiere? Mém. Acad. St. Pétersbg., Tme XIX No. 8, p. 2, 1873.

2. A. Kowalersky: Entwickinngsgeschichte der einfachen Ascidien. Mém. Acad. St. Pétersbg., VII. Série, Tme X, No. 15, p. 3, 1866.

3. C. v. Kupffer: Die Stammesverwandtschaft zwischen Ascidien und W rbeltieren. Arch. mikrosk. Anat., Bd. 6, Heft II, p. 126, 1870.

4. L. Chabry: Contribution à l'embryologie normale et tératologique des ascidies simples. Journ. Anat. Physiol., Paris 1887, p. 194 u. 170.

5 W. E. Castle: The early embryology of Ciona intestinalis. Bull. Mus. Comparat. Zoology, Harvard College, Vol. XXVII, 1895-96.

6. R. Hartmeyer: Die Ascidien der Arktis in: Römer und Schaudinn, Fauna arctica, v. 3 Lfg. 2, Jena 1903. 